\title{
A limit theorem for Bernoulli convolutions and the $\Phi$-variation of functions in the Takagi class
}

\author{
Xiyue Han*
}

\author{
Alexander Schied**
}

December 12, 2021

\author{
Zhenyuan Zhang§
}

\begin{abstract}
We consider a probabilistic approach to compute the Wiener-Young $\Phi$-variation of fractal functions in the Takagi class. Here, the $\Phi$-variation is understood as a generalization of the quadratic variation or, more generally, the $p^{\text {th }}$ variation of a trajectory computed along the sequence of dyadic partitions of the unit interval. The functions $\Phi$ we consider form a very wide class of functions that are regularly varying at zero. Moreover, for each such function $\Phi$, our results provide in a straightforward manner a large and tractable class of functions that have nontrivial and linear $\Phi$-variation. As a corollary, we also construct stochastic processes whose sample paths have nontrivial, deterministic, and linear $\Phi$-variation for each function $\Phi$ from our class. The proof of our main result relies on a limit theorem for certain sums of Bernoulli random variables that converge to an infinite Bernoulli convolution.
\end{abstract}

Key words: Wiener-Young $\Phi$-variation, Takagi class, pathwise Itô calculus, infinite Bernoulli convolution, central limit theorem, stochastic process with prescribed $\Phi$-variation

MSC 2020: 60F 25, 28A80, 26A12, 60H05

\section{Introduction}

Probabilistic models of continuous random trajectories typically rely on solutions to stochastic differential equations or, more generally, on continuous semimartingales. The huge success of those models is in no small part due to the fact that they can be analyzed by means of Itô calculus. Itô calculus is however not restricted to the sample paths of a semimartingale: Föllmer [8] showed that every continuous function that admits a continuous quadratic variation along a fixed refining sequence of partitions can be used as an integrator for Itô integration and that a corresponding Itô formula holds. As shown in $[10,20,24]$, this includes many functions previously studied in fractal analysis and geometry.

Cont and Perkowski [4] recently extended Föllmer's pathwise Itô formula to integrators with higher than quadratic variation, the so-called $p^{\text {th }}$ variation. While quadratic variation is based on the squared increments of a function, $p^{\text {th }}$ variation is based on the $p^{\text {th }}$ power of the increments, where $p \geq 1$. In the realm of stochastic processes, the sample paths of fractional Brownian motion with Hurst index $1 / p$ constitute the best-known class of such trajectories. At the same time, recent applications, such as the

*Department of Statistics and Actuarial Science, University of Waterloo. E-mail: xiyue.han@uwaterloo.ca

${ }^{* *}$ Department of Statistics and Actuarial Science, University of Waterloo. E-mail: aschied@uwaterloo.ca

${ }^{\S}$ Department of Mathematics, Stanford University. E-mail: zzy@stanford.edu

The authors gratefully acknowledge financial support from the Natural Sciences and Engineering Research Council of Canada through grant RGPIN-2017-04054 
theory of rough volatility models based on the seminal paper [11] by Gatheral et al., have intensified the interest in creating new models for "rough" phenomena beyond the case of quadratic variation. Also in this strand of literature, the focus has so far been on models with finite and nontrivial $p^{\text {th }}$ variation.

From a mathematical point of view, however, there is no reason why attention should be restricted to variation based on power functions. A natural and much broader concept is given by the notion of $\Phi$-variation in the Wiener-Young sense. Here, for a function $\Phi:[0, \infty) \rightarrow[0, \infty)$, the $\Phi$-variation of a continuous function $f:[0,1] \rightarrow \mathbb{R}$ along the dyadic partitions is given by

$$
\langle f\rangle_{t}^{\Phi}:=\lim _{n \uparrow \infty} \sum_{k=0}^{\left\lfloor t 2^{n}\right\rfloor} \Phi\left(\left|f\left((k+1) 2^{-n}\right)-f\left(k 2^{-n}\right)\right|\right), \quad 0 \leq t \leq 1,
$$

provided that the limit exists for all $t$. Yet, most authors still focus exclusively on the case of power variation. Notable exceptions are the paper [18] by Marcus and Rosen, in which the $\Phi$-variation of Gaussian processes with stationary increments and local times of symmetric Lévy processes is obtained, and Kôno [16]. Note that the $\Phi$-variation along a fixed refining sequence of partitions as defined in (1.1) typically differs from the $\Phi$-variation defined as a supremum taken over all finite partitions. For Gaussian processes, the latter concept was studied, e.g., by Taylor [27] for $\Phi(x)=x^{2}$ and by Kawada and Kôno [15] for more general functions $\Phi$.

In this paper, we use a probabilistic approach to explore a class of fractal trajectories $f$ that admit the $\Phi$-variation $\langle f\rangle_{t}^{\Phi}=t$ for functions $\Phi$ of the form

$$
\Phi(x):= \begin{cases}0 & \text { if } x=0, \\ x^{p} g\left(-p \log _{2} x\right)^{-p / 2} & \text { if } 0<x<1,\end{cases}
$$

where $p \in[1, \infty)$ and $g:[0, \infty) \rightarrow(0, \infty)$ is any regularly varying function ${ }^{1}$. In doing so, we address several high-level issues. First, we illustrate that finite and nontrivial $p^{\text {th }}$ power variation, which has so far been the default paradigm for characterizing the roughness of trajectories, appears to be the exception rather than the rule. In particular, one should expect $\Phi$-variation rather than power variation when studying continuous trajectories that do not arise as sample paths of a semimartingale. Second, for every function $\Phi$ of the form (1.2), we construct explicit examples of trajectories with finite linear $\Phi$ variation along the dyadic partitions. These examples can be used in further analyses involving "rough" trajectories. Moreover, the idea underlying our construction of functions with prescribed $\Phi$-variation can probably be extended to more flexible models and into the realm of stochastic processes.

The class of functions $f$ we consider here belong to the so-called Takagi class, which was introduced by Hata and Yamaguti [14] and motivated by Takagi's [26] celebrated example of a continuous nowhere differentiable function. This class was chosen due to its richness and tractability and also because it is closely related to the Wiener-Lévy construction of Brownian motion. The Takagi class consists of all functions $f:[0,1] \rightarrow \mathbb{R}$ that admit an expansion of the form

$$
f(t):=\sum_{m=0}^{\infty} \alpha_{m} \varphi\left(2^{m} t\right), \quad t \in[0,1]
$$

where $\varphi(t)=\min _{z \in \mathbb{Z}}|z-t|$ is the tent map and $\left\{\alpha_{m}\right\}_{m \in \mathbb{N}_{0}}$ is a sequence of real numbers for which the series $\sum_{m=0}^{\infty} \alpha_{m}$ converges absolutely. The special choice $\alpha_{m}=a^{m}$ for some $a \in(-1,1)$ yields the

\footnotetext{
${ }^{1}$ Recall that a measurable and strictly positive function $g$, which is defined on some interval $[a, \infty)$, is called regularly varying (at infinity) with index $\varrho \in \mathbb{R}$ if $g(\lambda x) / g(x) \rightarrow \lambda^{\varrho}$ as $x \uparrow \infty$ for all $\lambda>0$. If $\varrho=0$, the function $g$ is also called slowly varying. Throughout this paper, we will always assume that all regularly varying functions are defined on $[0, \infty)$. This assumption can be made without loss of generality, as one can always consider the function $x \mapsto g(a \vee x)$.
} 
class of so-called Takagi-Landsberg functions, which, for $1 / 2<|a|<1$ and $p=-\log _{|a|} 2$, have finite nontrivial and linear $p^{\text {th }}$ variation as shown in [21]. For $|a|<1 / 2$ it is well known that $f$ is of finite total variation. The borderline case $a=1 / 2$ corresponds to the classical Takagi function, which is nowhere differentiable and hence not of finite total variation, even though $p=-\log _{1 / 2} 2=1$. Instead, for $|a|=1 / 2$, the function $f$ has linear $\Phi$-variation, $\langle f\rangle_{t}^{\Phi}=t$, for $\Phi(x)=x \sqrt{\pi /\left(-2 \log _{2} x\right)}$, as shown by the authors in [13]. Note that this function $\Phi$ is a special case of (1.2). Our method also extends to an even richer class of functions for which each scaled phase of the tent map in (1.3) is multiplied with an arbitrary sign (see (3.7) for details). This latter class can be fitted to empirical time series and gives also rise to a class of stochastic processes if the signs are chosen randomly.

Our approach to the $\Phi$-variation of functions $f$ in the Takagi class depends on the parameter $p$ in (1.2). For $p=1$, our argument extends the approach via the central limit theorem for the Rademacher functions in the Faber-Schauder development of $f$ as presented in [13]. For $p>1$, however, a new limit theorem is needed, which might be of independent interest. If $\left\{\beta_{m}\right\}_{m \in \mathbb{N}_{0}}$ is a sequence of real numbers and $Y_{1}, Y_{2}, \ldots$ is an i.i.d. sequence of symmetric Bernoulli random variables, we investigate the limit of the "convolutions"

$$
\frac{1}{s_{n}} \sum_{m=1}^{n} \beta_{n-m} Y_{m} \quad \text { where } \quad s_{n}^{2}:=\sum_{m=0}^{n-1} \beta_{m}^{2} .
$$

We show that if there exist $q>0, b>1$, and a slowly varying function $\ell$ such that

$$
\lim _{n \rightarrow \infty} \frac{s_{n}^{2}}{2^{2 q n} \ell\left(b^{n}\right)}=1
$$

then

$$
\frac{1}{s_{n}} \sum_{m=1}^{n} \beta_{n-m} Y_{m} \longrightarrow \sqrt{1-2^{-2 q}} \sum_{m=1}^{\infty} 2^{-q m} Y_{m} \quad \text { in } L^{\infty} .
$$

Note that the law of the limiting random variable $Z$ is a scaled version of the infinite Bernoulli convolution with parameter $2^{-q}$. In Section 2, we will present an extended version of this result in which the $Y_{m}$ are neither required to be independent nor identically distributed. This approach to the $\Phi$-variation via the condition (1.4) and the limiting result (1.5) is to some extent motivated by Gladyshev's theorem [12] (see also the book [19] by Marcus and Rosen for a comprehensive survey). It extends previous work in $[21,25,13]$.

The paper is structured as follows. In Section 2, we derive the convergence result (1.5) and the central limit theorem that will be needed for our results on the $\Phi$-variation of functions in the Takagi class. Our main result on this topic, Theorem 3.3, is stated in Section 3. The proof of Theorem 3.3 is given in Section 4.

\section{A limit theorem for Bernoulli-type convolutions}

For some $p \in[1, \infty]$, let $Y_{1}, Y_{2}, .$. be a sequence of random variables in $L^{p}:=L^{p}(\Omega, \mathscr{F}, \mathbb{P})$, where $(\Omega, \mathscr{F}, \mathbb{P})$ is a given probability space. We assume for simplicity that the $L^{p}$-norms of $Y_{m}$ are uniformly bounded. Let furthermore $\left\{\beta_{m}\right\}_{m \in \mathbb{N}_{0}}$ be a sequence of real numbers and consider the random variables

$$
Z_{n}:=\sum_{m=1}^{n} \beta_{n-m} Y_{m}, \quad n \in \mathbb{N}
$$

We are first concerned with the limit of

$$
\frac{1}{s_{n}} Z_{n} \quad \text { where } \quad s_{n}^{2}:=\sum_{m=0}^{n-1} \beta_{m}^{2}
$$


as $n \uparrow \infty$. Our corresponding result uses the concept of a slowly varying function, which was recalled in the footnote on page 2 .

Theorem 2.1. Suppose that $\beta_{m} \geq 0$ for all $m$ and that there exist $q>0, b>1$, and a slowly varying function $\ell$ such that

$$
\lim _{n \rightarrow \infty} \frac{s_{n}^{2}}{2^{2 q n} \ell\left(b^{n}\right)}=1
$$

Then

$$
\frac{1}{s_{n}} Z_{n} \longrightarrow Z:=\sqrt{2^{2 q}-1} \sum_{m=1}^{\infty} 2^{-q m} Y_{m} \quad \text { in } L^{p}
$$

Proof of Theorem 2.1. For $\delta \in\left(0, \delta_{0}\right]$, denote

$$
\lambda:=\sqrt{2^{2 q}-1}, \quad \lambda_{\delta}^{+}:=\sqrt{2^{2 q} \frac{(1+\delta)^{2}}{1-\delta}-1}, \quad \text { and } \quad \lambda_{\delta}^{-}:=\sqrt{2^{2 q} \frac{(1-\delta)^{2}}{1+\delta}-1}
$$

where $\delta_{0} \in(0, \min \{1, q\})$ is chosen such that the arguments of the square roots are always positive. Thus,

$$
2^{2 q}(1-\delta)^{2}-(1+\delta)>0 \quad \text { for all } \delta \in\left(0, \delta_{0}\right] .
$$

Let $\varepsilon>0$ be given. We first choose $K \in \mathbb{N}$ such that

$$
\lambda \sum_{m=K+1}^{\infty} 2^{-\left(q-\delta_{0}\right) m}<\varepsilon
$$

Then we choose $\delta_{1} \in\left(0, \delta_{0}\right]$ such that

$$
1-2^{-\delta m}<\varepsilon \quad \text { for } m=0, \ldots, K \text { and all } \delta \in\left(0, \delta_{1}\right] .
$$

Finally, we choose $\delta \in\left(0, \delta_{1}\right]$ such that

$$
\left|\frac{\lambda_{\delta}^{ \pm}}{\sqrt{1 \mp \delta}}-\lambda\right|<\varepsilon
$$

Given this $\delta$, we use (2.1) to pick $n_{0} \in \mathbb{N}$ such that

$$
\ell\left(b^{n}\right) 2^{2 q n}(1-\delta)<s_{n}^{2}<\ell\left(b^{n}\right) 2^{2 q n}(1+\delta) \quad \text { for all } n>n_{0} .
$$

Hence,

$$
2^{2 q(m+1)}\left((1-\delta) \ell\left(b^{m+1}\right)-(1+\delta) \ell\left(b^{m}\right) 2^{-2 q}\right)<\beta_{m}^{2}<2^{2 q(m+1)}\left((1+\delta) \ell\left(b^{m+1}\right)-(1-\delta) \ell\left(b^{m}\right) 2^{-2 q}\right) .
$$

Since $\ell$ is slowly varying, we can choose $n_{1} \geq n_{0}$ such that

$$
1-\delta<\frac{\ell\left(b \cdot b^{m}\right)}{\ell\left(b^{m}\right)}<1+\delta \quad \text { for all } m \geq n_{1} .
$$

Combining this with (2.2), we see that the left-hand side of (2.7) is strictly positive for $m \geq n_{1}$. Hence, we may take square roots to get

$$
2^{q(m+1)} \sqrt{(1-\delta) \ell\left(b^{m+1}\right)-(1+\delta) \ell\left(b^{m}\right) 2^{-2 q}}<\beta_{m}<2^{q(m+1)} \sqrt{(1+\delta) \ell\left(b^{m+1}\right)-(1-\delta) \ell\left(b^{m}\right) 2^{-2 q}}
$$


for all $m \geq n_{1}$. Moreover, with (2.6) we get that for $n>m \geq n_{1}$,

$$
\begin{aligned}
\frac{\beta_{m}}{s_{n}} & <\frac{2^{q(m+1)} \sqrt{(1+\delta) \ell\left(b^{m+1}\right)-(1-\delta) \ell\left(b^{m}\right) 2^{-2 q}}}{\sqrt{(1-\delta) 2^{2 q n} \ell\left(b^{n}\right)}}=2^{q(m-n)} \sqrt{\frac{\ell\left(b^{m}\right)}{\ell\left(b^{n}\right)}\left(2^{2 q} \frac{1+\delta}{1-\delta} \frac{\ell\left(b^{m+1}\right)}{\ell\left(b^{m}\right)}-1\right)} \\
& <2^{q(m-n)} \sqrt{\frac{\ell\left(b^{m}\right)}{\ell\left(b^{n}\right)}} \lambda_{\delta}^{+} .
\end{aligned}
$$

Similarly, we conclude a corresponding lower bound and obtain that for $n>m \geq n_{1}$,

$$
2^{-q(n-m)} \sqrt{\frac{\ell\left(b^{m}\right)}{\ell\left(b^{n}\right)}} \lambda_{\delta}^{-}<\frac{\beta_{m}}{s_{n}}<2^{-q(n-m)} \sqrt{\frac{\ell\left(b^{m}\right)}{\ell\left(b^{n}\right)}} \lambda_{\delta}^{+} .
$$

The Potter bounds (see, e.g., Theorem 1.5.6 in [3]) yield that there is $n_{2} \geq n_{1}$ such that for $n \geq m \geq n_{2}$

$$
(1-\delta) 2^{-2 \delta(n-m)}=(1-\delta) b^{-2 \delta(n-m) \log _{b} 2} \leq \frac{\ell\left(b^{n}\right)}{\ell\left(b^{m}\right)} \leq(1+\delta) b^{2 \delta(n-m) \log _{b} 2}=(1+\delta) 2^{2 \delta(n-m)} .
$$

With (2.9), we obtain that for $n \geq n_{2}+m$,

$$
2^{-(q+\delta) m} \frac{\lambda_{\delta}^{-}}{\sqrt{1+\delta}}<\frac{\beta_{n-m}}{s_{n}}<2^{-(q-\delta) m} \frac{\lambda_{\delta}^{+}}{\sqrt{1-\delta}}
$$

Note that

$$
Z^{(n)}:=\lambda \sum_{m=1}^{n} 2^{-q m} Y_{m} \longrightarrow Z \quad \text { in } L^{p}
$$

By Minkowski's inequality and with $c:=\sup _{m}\left\|Y_{m}\right\|_{p}<\infty$,

$$
\left\|\frac{Z_{n}}{s_{n}}-Z^{(n)}\right\|_{p} \leq \sum_{m=1}^{n}\left|\frac{\beta_{n-m}}{s_{n}}-\lambda 2^{-q m}\right|\left\|Y_{m}\right\|_{p} \leq c \sum_{m=1}^{n-n_{2}}\left|\frac{\beta_{n-m}}{s_{n}}-\lambda 2^{-q m}\right|+c \sum_{m=n-n_{2}+1}^{n}\left|\frac{\beta_{n-m}}{s_{n}}-\lambda 2^{-q m}\right| .
$$

To deal with the first sum on the right-hand side, we use (2.5) and (2.10) to get that for $m \leq n-n_{2}$,

$$
\begin{aligned}
\frac{\beta_{n-m}}{s_{n}}-\lambda 2^{-q m} & \leq 2^{-(q-\delta) m}\left(\frac{\lambda_{\delta}^{+}}{\sqrt{1-\delta}}-\lambda\right)+\lambda\left(2^{-(q-\delta) m}-2^{-q m}\right) \\
& \leq \varepsilon 2^{-(q-\delta) m}+\lambda 2^{-(q-\delta) m}\left(1-2^{-\delta m}\right) .
\end{aligned}
$$

In the same way, we get the lower bound

$$
-\varepsilon 2^{-(q+\delta) m}-\lambda 2^{-q m}\left(1-2^{-\delta m}\right) \leq \frac{\beta_{n-m}}{s_{n}}-\lambda 2^{-q m} .
$$

Using (2.4) and (2.3), we thus get the following estimate for the first sum on the right-hand side of $(2.11)$

$$
\sum_{m=1}^{n-n_{2}}\left|\frac{\beta_{n-m}}{s_{n}}-\lambda 2^{-q m}\right| \leq \varepsilon \sum_{m=1}^{n-n_{2}} 2^{-(q-\delta) m}+\lambda \sum_{m=1}^{K} 2^{-(q-\delta) m} \varepsilon+\lambda \sum_{m=K+1}^{\infty} 2^{-(q-\delta) m} \leq \varepsilon c^{\prime}
$$


where $c^{\prime}:=2 \sum_{m=0}^{\infty} 2^{-\left(q-\delta_{0}\right) m}+1$. For the right-most sum in $(2.11)$, we have

$$
\begin{aligned}
\sum_{m=n-n_{2}+1}^{n}\left|\frac{\beta_{n-m}}{s_{n}}-\lambda 2^{-q m}\right| & \leq \sum_{m=n-n_{2}+1}^{n} \frac{\beta_{n-m}}{s_{n}}+\lambda \sum_{m=n-n_{2}+1}^{n} 2^{-q m} \\
& \leq \frac{n_{2}}{s_{n}} \max _{i=0, \ldots, n_{2}-1} \beta_{i}+\lambda 2^{-q n} \frac{2^{q n_{2}}-1}{2^{q}-1} .
\end{aligned}
$$

We will show below that our assumptions imply that $s_{n} \rightarrow \infty$. Therefore, the right-hand side of (2.12) can be made smaller than $\varepsilon$ by choosing $n$ sufficiently large. Thus, we see that the $L^{p}$-distance between $Z_{n} / s_{n}$ and $Z^{(n)}$ is smaller than $c\left(c^{\prime}+1\right) \varepsilon$ if $n$ is sufficiently large.

Finally, we argue that $s_{n} \rightarrow \infty$ if $(2.1)$ holds and $q>0$. To this end, let $g(x):=x^{2 q} \ell(x)$, so that the denominator in $(2.1)$ is equal to $g\left(2^{n}\right)$. Proposition 1.3.6 in [3] states that $g(x) \rightarrow \infty$, which in view of (2.1) yields our claim.

Now we briefly discuss the case where $q=0$ in (2.1). In that case, we are typically in the regime of the central limit theorem. This is suggested, for instance, by [28], where a central limit theorem for reversible Markov chains was obtained under the condition that the variance, $\sigma_{n}^{2}$, satisfies $\sigma_{n}^{2}=n \ell(n)$ for a slowly varying function $\ell$. Since $g(x):=x \ell(x)$ is regularly varying with index $\varrho=1$, we see that, in our situation, this condition is a special case of condition (3.4) with $q=0$. In the following proposition, we formulate implications between stronger and weaker versions of condition (2.1) with $q=0$.

Now we are going to formulate a simple central limit theorem in the form needed in the next section. We believe that this result is well known but were unable to find an exact reference to the literature. Due to the large number of existing variants of the central limit theorem, we confine ourselves here to the basic case that will actually be needed in Section 4 .

Lemma 2.2. Suppose that $Y_{1}, Y_{2}, \ldots$ is an i.i.d. sequence of symmetric $\{-1,+1\}$-valued Bernoulli random variables such that $s_{n-1}^{2} / s_{n}^{2} \rightarrow 1$ as $n \uparrow \infty$. Then, for every $p \in[1, \infty)$, the laws of $\frac{1}{s_{n}} Z_{n}$ converge in the $L^{p}$-Wasserstein metric to the standard normal distribution.

Proof. As $s_{n-1}^{2} / s_{n}^{2} \rightarrow 1$, it then follows that

$$
\lim _{n \uparrow \infty} \frac{\beta_{n}^{2}}{s_{n}^{2}}=\lim _{n \uparrow \infty}\left(1-\frac{s_{n-1}^{2}}{s_{n}^{2}}\right)=0 .
$$

It is hence easy to see that the sequence $\left\{Z_{n}\right\}_{n \in \mathbb{N}_{0}}$ satisfies Lindeberg's condition, which yields that $\frac{1}{s_{n}} Z_{n}$ converges in law to $N(0,1)$. Thus, to conclude convergence in the $L^{p}$-Wasserstein metric, it is sufficient to show that $\mathbb{E}\left[\left|s_{n}^{-1} Z_{n}\right|^{q}\right]$ is uniformly bounded in $n$ for some $q>p$; this follows, e.g., from Lemma 5.61 and Corollary A.50 in [9]. We actually show a stronger condition, namely that $\mathbb{E}\left[e^{\left|\lambda Z_{n} / s_{n}\right|}\right]$ is uniformly bounded for all $\lambda \in \mathbb{R}$. Since $e^{|x|} \leq e^{x}+e^{-x}$, we can drop the absolute value in the exponent. Then,

$$
\log \mathbb{E}\left[e^{\lambda Z_{n} / s_{n}}\right]=\log \prod_{k=0}^{n} \cosh \left(\lambda \beta_{k} / s_{n}\right)=\sum_{k=0}^{n} \log \cosh \left(\lambda \beta_{k} / s_{n}\right) \leq \sum_{k=0}^{n} \frac{\lambda^{2} \beta_{k}^{2}}{2 s_{n}^{2}}=\frac{\lambda^{2}}{2} .
$$

\section{$3 \quad \Phi$-variation of functions in the Takagi class}

In this section, we will apply the limit theorems from Section 2 to the problem of computing the $\Phi$-variation of functions in the Takagi class. To this end, let $\Phi:[0, \infty) \rightarrow[0, \infty)$ be a function with 
$\Phi(0)=0$. The $\Phi$-variation of a continuous function $f:[0,1] \rightarrow \mathbb{R}$ along the sequence of dyadic partitions is defined as

$$
\langle f\rangle_{t}^{\Phi}:=\lim _{n \uparrow \infty} \sum_{k=0}^{\left\lfloor t 2^{n}\right\rfloor} \Phi\left(\left|f\left((k+1) 2^{-n}\right)-f\left(k 2^{-n}\right)\right|\right), \quad 0 \leq t \leq 1,
$$

provided that the limit exists for all $t \in[0,1]$. Due to the uniform continuity of $f$, we may, without loss of generality, restrict $\Phi$ to the domain $[0,1)$. For $\Phi(x)=x$ and $t=1$, we obtain the total variation of $f$. For $\Phi(x)=x^{2}$, we obtain the usual quadratic variation, and for $\Phi(x)=x^{p}$ with $p \geq 1$ the $p^{\text {th }}$ variation. Here, we are interested in functions for which the correct $\Phi$ may not be of the form $\Phi(x)=x^{p}$ but rather has a more complex structure.

Let $\varphi(t)=\min _{z \in \mathbb{Z}}|z-t|$ denote the tent map. The Takagi class, as introduced by Hata and Yamaguti [14], consists of all functions $f:[0,1] \rightarrow \mathbb{R}$ that admit an expansion of the form

$$
f(t):=\sum_{m=0}^{\infty} \alpha_{m} \varphi\left(2^{m} t\right), \quad t \in[0,1]
$$

where $\left\{\alpha_{m}\right\}_{m \in \mathbb{N}_{0}}$ is a sequence of real numbers for which the series $\sum_{m=0}^{\infty} \alpha_{m}$ converges absolutely. Clearly, the series on the right-hand side of (3.1) converges uniformly in $t \in[0,1]$, so that $f$ is indeed a well defined continuous function. Typically, the functions in the Takagi class have a fractal structure. For instance, the choice $\alpha_{m}=2^{-m}$ corresponds to the classical, nowhere differentiable Takagi function, which was originally introduced in [26] but rediscovered many times. More generally, the choice $\alpha_{m}=$ $a^{m}$ for some $a \in(-1,1)$ yields the class of so-called Takagi-Landsberg functions. Analytical properties of the functions in the Takagi class, such as differentiability, are discussed in [17].

In our first result, we take $f$ as in (3.1) as given and relate the asymptotic behavior of

$$
V_{n}^{(p)}:=\sum_{k=0}^{2^{n}-1}\left|f\left((k+1) 2^{-n}\right)-f\left(k 2^{-n}\right)\right|^{p}, \quad p \geq 1, n \in \mathbb{N},
$$

to the one of

$$
s_{n}^{2}:=\sum_{m=0}^{n-1} \alpha_{m}^{2} 4^{m}, \quad n \in \mathbb{N} .
$$

Note that $V_{n}^{(1)}$ converges to the total variation of $f$, defined as usual through taking the supremum over all possible partitions of $[0,1]$ (see, e.g., Theorem 2 in $\S 5$ of Chapter VIII in [22]). The following proposition provides a priori bounds on the behavior of $V_{n}^{(p)}$, based on the range of possible growth rates of $s_{n}$.

Proposition 3.1. Let

$$
q_{*}:=\liminf _{n \uparrow \infty} \frac{1}{n} \log _{2} s_{n} \quad \text { and } \quad q^{*}:=\limsup _{n \uparrow \infty} \frac{1}{n} \log _{2} s_{n} .
$$

Then, for $p \geq 1$, we have $\liminf _{n} V_{n}^{(p)}=0$ if $p\left(1-q_{*}\right)>1$ and $\lim \sup _{n} V_{n}^{(p)}=\infty$ if $p\left(1-q^{*}\right)<1$.

Example 3.2. Faber [7] considered the function $f$ with

$$
\alpha_{m}= \begin{cases}\frac{1}{10^{n}} & \text { if } m=n ! \text { for some } n \in \mathbb{N}, \\ 0 & \text { otherwise, }\end{cases}
$$

and showed that it is nowhere differentiable. It is easy to see that this choice leads to $q^{*} \geq 1$ and $q_{*} \leq 0$, which implies that $\liminf V_{n} V_{n}^{(p)}=0$ and $\lim \sup _{n} V_{n}^{(p)}=\infty$ for all $p \geq 1$. 
A more interesting case is the situation in which $f$ is such that $q:=\lim _{n} \frac{1}{n} \log _{2} s_{n}$ exists and satisfies $0 \leq q<1$. Then Proposition 3.1 implies that $p:=1 /(1-q)$ is the critical exponent for the power variation of $f$. The standard paradigm in the literature is to expect that $f$ then admits a finite $p^{\text {th }}$ variation. This is true for the typical sample paths of a continuous semimartingale with $p=2$ and for the trajectories of fractional Brownian motion with Hurst exponent $H=1 / p$. As shown in [21, 25], it is also true for the Takagi-Landsberg functions. In the sequel, we shall in particular investigate the situation in which $\lim _{n} V_{n}^{(p)}$ exists but is either zero or infinity. In this case, $p^{\text {th }}$ power variation is clearly no longer sufficient for characterizing the exact "roughness" of $f$.

To state our first main result, we recall that Corollary 1.4.2 in [3] allows us to assume without loss of generality that all regularly varying functions are bounded on compact intervals (see the footnote on page 2 for the definition of a regularly varying function). If $g$ is regularly varying and $q \in[0,1)$, consider

$$
\Phi_{q}(x)=\Phi_{q, g}(x):=x^{\frac{1}{1-q}} g\left(\frac{-\log _{2} x}{1-q}\right)^{-\frac{1}{2(1-q)}}
$$

for $0<x<1$. By Proposition 1.5.7 in [3], $y \mapsto g\left(\left(\log _{2} y\right) /(1-q)\right)$ is slowly varying, and so $\psi_{q}(y):=$ $\Phi_{q}(1 / y)$ is regularly varying with index $-1 /(1-q)$. We conclude that $\Phi_{q}(x) \rightarrow 0$ as $x \downarrow 0$. It therefore makes sense to extend $\Phi_{q}$ to the domain $[0,1)$ by setting $\Phi_{q}(0):=0$. Note also that $\Phi_{q}$ is slowly varying at zero with index $1 /(1-q)$ and that the functions arising in this manner form a very wide class of functions that are regularly varying at zero.

Theorem 3.3. For $f$ as in (3.1), let $s_{n}^{2}$ be as in (3.2) and suppose that there exist $q \in[0,1)$ and a regularly varying function $g$ such that

$$
\lim _{n \uparrow \infty} \frac{s_{n}^{2}}{2^{2 q n} g(n)}=1 \text {. }
$$

Let moreover $\left\{Y_{m}\right\}_{m \in \mathbb{N}}$ be an i.i.d. sequence of symmetric $\{-1,+1\}$-valued Bernoulli random variables and $\Phi_{q}$ as in (3.3). Then the following hold:

(a) The function $f$ is of bounded variation if and only if $\lim _{n} s_{n}^{2}<\infty$ and, in this case, the total variation of $f$ is equal to $\mathbb{E}[|\widetilde{Z}|]$, where $\widetilde{Z}:=\sum_{m=0}^{\infty} \alpha_{m} 2^{m} Y_{m+1}$.

(b) If $s_{n}^{2} \rightarrow \infty$ and $q=0$, then the $\Phi_{0}$-variation of $f$ is given by

$$
\langle f\rangle_{t}^{\Phi_{0}}=\sqrt{\frac{2}{\pi}} \cdot t
$$

(c) If $q>0$, then the $\Phi_{q}$-variation of $f$ is given by

$$
\langle f\rangle_{t}^{\Phi_{q}}=\mathbb{E}\left[|Z|^{\frac{1}{1-q}}\right] \cdot t \quad \text { where } \quad Z:=\sqrt{2^{2 q}-1} \sum_{m=1}^{\infty} 2^{-q m} Y_{m}
$$

Remark 3.4. The random variable $Z$ in (3.6) can be represented as

$$
Z=\sqrt{1-2^{-2 q}} \sum_{m=0}^{\infty} 2^{-q m} Y_{m+1}
$$

and hence has as its law a scaled version of the infinite Bernoulli convolution with parameter $2^{-q}$. These laws exhibit some fascinating properties and have been studied in their own right for many decades; see, e.g., [23] and the references therein. 
In the next two results, we consider again the situation of Proposition 3.1 and take a closer look at what happens to the power variation of a function $f$ in the Takagi class satisfying (3.4).

Corollary 3.5. Suppose that (3.4) holds with $q \in[0,1)$ and let $p:=1 /(1-q)$.

(a) The function $f$ has infinite $r^{\text {th }}$ variation for $1 \leq r<p$ and vanishing $r^{\text {th }}$ variation for $r>p$.

(b) If $\lim _{n} g(n)=c$ for some number $c \in[0, \infty]$, then the $p^{\text {th }}$ variation of $f$ satisfies $\langle f\rangle_{t}^{(p)}=c^{p / 2}\langle f\rangle_{t}^{\Phi_{q}}$ for $t \in[0,1]$.

If $g$ is regularly varying with index $\varrho \in \mathbb{R}$, then $g(x) \rightarrow 0$ for $\varrho<0$ and $g(x) \rightarrow \infty$ for $\varrho>0$ according to Proposition B.1.9 (1) in [5]. If $\varrho=0$, then $g$ is slowly varying and may or may not converge to a number $c \in[0, \infty]$. In conjunction with Corollary 3.5, we thus get immediately the following corollary.

Corollary 3.6. Suppose that (3.4) holds with $q \in[0,1)$ and a function $g$ that is regularly varying with index $\varrho \in \mathbb{R}$. We let $p:=1 /(1-q)$.

(a) If $\varrho<0$, then $f$ has vanishing $p^{\text {th }}$ variation.

(b) If $\varrho>0$, then $f$ has infinite $p^{\text {th }}$ variation.

Example 3.7. Let us consider the classical Takagi function,

$$
f(t)=\sum_{m=0}^{\infty} 2^{-m} \varphi\left(2^{m} t\right)
$$

as discussed in the introduction. Then $s_{n}^{2}=n$ and so we can take $q=0$ and $g(x):=x$. Corollary 3.6 thus immediately yields the well-known fact that $f$ is of unbounded total variation. Moreover, we have $\Phi_{0}(t)=x / \sqrt{-\log _{2} x}$, and so we recover here a special case of Theorem 1.2 in [13].

Allaart [1] introduced an extension of the Takagi class by multiplying each replicate of the tent map in the expansion (3.1) with an arbitrary sign. More precisely, he considered the class of functions of the form

$$
f(t):=\sum_{m=0}^{\infty} \alpha_{m} \sigma_{m}(t) \varphi\left(2^{m} t\right), \quad t \in[0,1],
$$

where $\sigma_{m}:[0,1] \rightarrow\{-1,+1\}$ is constant on each interval $\left[(k-1) 2^{-m}, k 2^{-m}\right)$ for $k=1, \ldots, 2^{m}$, and $\left\{\alpha_{m}\right\}_{m \in \mathbb{N}_{0}}$ is a sequence of real numbers for which the series $\sum_{m} \alpha_{m}$ converges absolutely. Then $f$ is well-defined and continuous due to the uniform convergence of the series in (3.7). Certain deterministic choices for $\sigma_{m}$ yield fractal functions studied in other fields such as information theory; see $[1,2]$ and the references therein. When the signs of $\sigma_{m}$ are chosen in a random manner, the expansion (3.7) becomes closely related to the Wiener-Lévy-Ciesielski expansion of Brownian motion by means of the Faber-Schauder functions, and the functions (3.7) with fixed coefficients $\left\{\alpha_{m}\right\}_{m \in \mathbb{N}_{0}}$ but random signs form a non-Gaussian stochastic process with rough sample paths. More precisely, for each $m$, let $\left\{\xi_{m, k}\right\}_{k=1, \cdots, 2^{m}}$ be a sequence of $\{-1,+1\}$-valued Bernoulli random variables and define

$$
\sigma_{m}(t):=\sum_{k=1}^{2^{m}} \xi_{m, k} \mathbb{1}_{\left[(k-1) 2^{-m}, k 2^{-m}\right)}(t) \quad \text { and } \quad X_{t}:=\sum_{m=0}^{\infty} \alpha_{m} \sigma_{m}(t) \varphi\left(2^{m} t\right) .
$$

Then the following corollary states that $\left\{X_{t}\right\}_{t \in[0,1]}$ is a stochastic process whose sample paths have the deterministic linear $\Phi_{q}$-variation (3.6) if the sequence $\left\{\alpha_{m}\right\}_{m \in \mathbb{N}_{0}}$ satisfies the conditions on Theorem 3.3. An illustration of this stochastic process is provided in Figure 1. For the choice $\alpha_{m}=a^{m}$ with $a \in(-1,1)$, the $p^{\text {th }}$ variation of the functions (3.7) was studied in $[21,24]$. 
Corollary 3.8. The statements of Theorem 3.3 and of the Corollaries 3.5 and 3.6 remain fully valid for the functions of the form (3.7).

When the goal is to utilize Theorem 3.3 for the construction of functions with prescribed $\Phi_{q^{-}}$ variation for a given function $g$, it can be inconvenient that the condition (3.4) is formulated in terms of the sums $s_{n}^{2}=\sum_{m=0}^{n-1} \alpha_{m}^{2} 4^{m}$ rather than in terms of the coefficients $\alpha_{m}$ themselves. To deal with this issue, we are going to formulate Proposition 3.10, which will also be used in the proofs of our main results.

Remark 3.9. Let us recall the following facts on slowly and regularly varying functions so as to put the statement of the following Proposition 3.10 into the context of Theorem 3.3.

(a) If $g$ is a regularly varying function and $b>1$, then $\ell(x):=g\left(\log _{b} x\right)$ is slowly varying according to Proposition 1.5.7 (ii) in [3]. Moreover, our condition (3.4) implies that

$$
\lim _{n \rightarrow \infty} \frac{s_{n}^{2}}{2^{2 q n} \ell\left(b^{n}\right)}=1 .
$$

(b) Recalling that we assume all regularly (and hence slowly) varying functions to be locally bounded, and thus locally integrable, we may define

$$
\ell(x):=\frac{1}{\log b} \int_{1}^{x} \frac{1}{t} L(t) d t=\int_{0}^{\log _{b} x} L\left(b^{s}\right) d s
$$

for a given slowly varying function $L$. Then, according to Proposition 1.5.9 (a) in [3], the function $\ell$ is slowly varying and satisfies $\ell(x) / L(x) \rightarrow+\infty$ as $x \uparrow \infty$.

Proposition 3.10. Let $\left\{\beta_{m}\right\}_{m \in \mathbb{N}_{0}}$ be a sequence of real numbers, $s_{n}^{2}=\sum_{m=0}^{n-1} \beta_{m}^{2}$, and $b>1$.

(a) Suppose that $s_{n} \rightarrow \infty$ as $n \uparrow \infty$, let $L$ be a slowly varying function, define $\ell$ as in (3.9), and consider the following two conditions:

(i) $\beta_{n}^{2} / L\left(b^{n}\right) \rightarrow 1$ as $n \uparrow \infty$.

(ii) $s_{n}^{2} / \ell\left(b^{n}\right) \rightarrow 1$ as $n \uparrow \infty$.

Then (i) $\Rightarrow($ ii) .

(b) If $\ell$ is a slowly varying function and $q>0$, the following are equivalent:

(iii) $\beta_{n}^{2} / 2^{2 q n} \ell\left(b^{n}\right) \rightarrow 1$ as $n \uparrow \infty$.

(iv) $s_{n}^{2} / 2^{2 q n} \ell\left(b^{n}\right) \rightarrow\left(2^{2 q}-1\right)^{-1}$ as $n \uparrow \infty$.

(c) Any of the conditions (i)-(iv) implies $s_{n-1}^{2} / s_{n}^{2} \rightarrow 2^{2 q}$ as $n \uparrow \infty$, where we take $q=0$ in the context of (a).

The proofs of Proposition 3.1, Theorem 3.3, Corollary 3.8, Corollary 3.5, and Proposition 3.10 are given in Section 4. Theorem 3.3 and Proposition 3.10 yield immediately the following result, which provides a simple construction for a function with prescribed $\Phi_{q}$-variation. When applying it to the stochastic process (3.8), it provides a straightforward way of constructing a stochastic process whose sample paths have deterministic and linear $\Phi_{q}$-variation; see Figure 1 for an illustration. 
Corollary 3.11. Let $g$ be a regularly varying function, $q \in[0,1)$, and $\Phi_{q}$ as in $(3.3)$.

(a) For $q \in(0,1)$, the functions $f$ in (3.1) and (3.7) with coefficients

$$
\alpha_{m}:=2^{-m(1-q)} \sqrt{\left(2^{2 q}-1\right) g(m)}
$$

have linear $\Phi_{q}$-variation (3.6).

(b) For $q=0$, we assume in addition that $g(x) \rightarrow \infty$ as $x \uparrow \infty$ and that $g$ is absolutely continuous with regularly varying derivative $g^{\prime}$. Then the functions $f$ in (3.1) and (3.7) with coefficients

$$
\alpha_{m}:=2^{-m} \sqrt{g^{\prime}(m)}
$$

have linear $\Phi_{q}$-variation $(3.5)$.

Proof. (b) Since the function $L(x):=g^{\prime}\left(\log _{2} x\right)$ is slowly varying by Remark 3.9, Proposition 3.10 (a) yields that $s_{n}^{2} / \ell\left(2^{n}\right) \rightarrow 1$, where $\ell(x)=\int_{0}^{\log _{2} x} L\left(2^{t}\right) d t=g\left(\log _{2} x\right)-g(0)$. Hence, the conditions of Theorem 3.3 (b) are satisfied. Part (a) is now obvious.
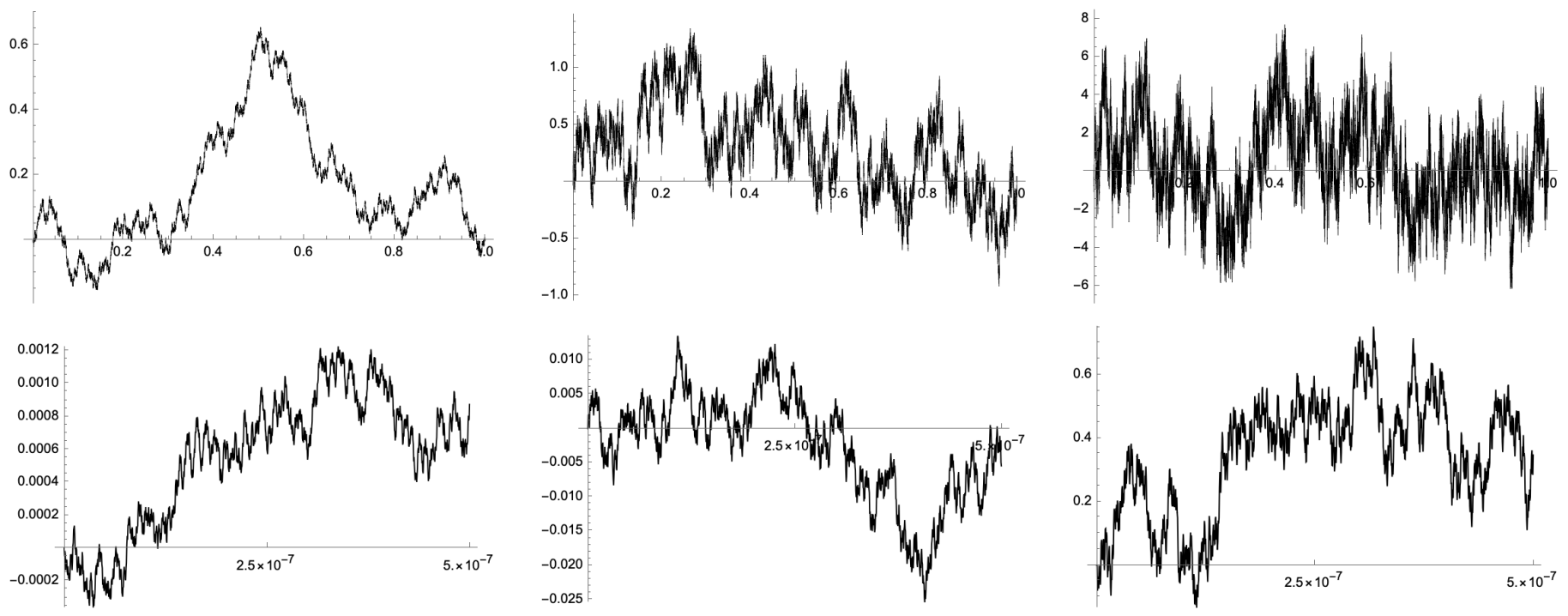

Figure 1: Sample paths of the stochastic process $\left\{X_{t}\right\}_{t \in[0,1]}$ defined in (3.8) with $\alpha_{m}=$ $2^{-m(1-q)} \sqrt{\left(2^{2 q}-1\right) g(m)}$ for $q=0.7, g(x)=(1+x)^{\varrho}$ with $\rho=-2$ (left), $\rho=0$ (center), $\rho=2$ (right), and signs $\left\{\xi_{m, k}\right\}_{m \in \mathbb{N}_{0}, k=1, \cdots, 2^{m}}$ that are i.i.d. $\{-1,+1\}$-valued Bernoulli random variables. For each $\varrho$, a new realization of $\left\{\xi_{m, k}\right\}$ was chosen. One can see from the three top panels that the parameter $\varrho$ has a strong influence on the macroscopic fluctuations of the trajectories. The fact that all three functions share the same critical exponent $p=1 /(1-q)=10 / 3$ as defined in Proposition 3.1 suggest a common Hurst exponent of $H=1 / p=0.3$ (see the discussion on page 8). One would thus expect the fluctuations of the three functions to be similar on a microscopic scale. This effect can indeed be observed in the lower panels below, which show the same three functions after zooming into the interval $[0, \varepsilon]$ for $\varepsilon=5 \cdot 10^{-7}$. 


\section{Proofs}

Proof of Proposition 3.10. (i) $\Rightarrow$ (ii): For any given $\varepsilon>0$, there exists $n_{0}$ such that for all $m>n_{0}$, we have $L\left(b^{m}\right)(1-\varepsilon)<\beta_{m}^{2}<L\left(b^{m}\right)(1+\varepsilon)$. Hence, for all $\nu \geq n_{0}$ and $n>\nu$,

$$
\sum_{m=0}^{\nu} \beta_{m}^{2}+(1-\varepsilon) \sum_{m=\nu+1}^{n-1} L\left(b^{m}\right)<\sum_{m=0}^{n-1} \beta_{m}^{2}<\sum_{m=0}^{\nu} \beta_{m}^{2}+(1+\varepsilon) \sum_{m=\nu+1}^{n-1} L\left(b^{m}\right) .
$$

The Potter bounds (e.g., Theorem 1.5.6 in [3]) yield for every $\delta>0$ some $n_{1} \in \mathbb{N}$ such that

$$
(1-\varepsilon) b^{\delta(m-t)} \leq \frac{L\left(b^{t}\right)}{L\left(b^{m}\right)} \leq(1+\varepsilon) b^{\delta(t-m)} \quad \text { for all } t, m \geq n_{1} .
$$

We hence get that for $m \geq n_{1}$,

$$
L\left(b^{m}\right)(1-\varepsilon) \frac{1-b^{-\delta}}{\delta \log b} \leq \int_{m}^{m+1} L\left(b^{t}\right) d t \leq L\left(b^{m}\right)(1+\varepsilon) \frac{b^{\delta}-1}{\delta \log b} .
$$

Since $\pm\left(1-b^{\mp \delta}\right) /(\delta \log b) \rightarrow 1$ as $\delta \downarrow 0$, we can choose $\delta$ small enough such that for any $m \geq n_{1}$,

$$
(1-\varepsilon)^{2} L\left(b^{m}\right) \leq \int_{m}^{m+1} L\left(b^{t}\right) d t \leq(1+\varepsilon)^{2} L\left(b^{m}\right) .
$$

Using this estimate in (4.1) and dividing both sides of the result by $\int_{0}^{n} L\left(b^{t}\right) d t=\ell\left(b^{n}\right)$ gives

$$
\frac{\sum_{m=0}^{n_{1}-1} \beta_{m}^{2}}{\int_{0}^{n} L\left(b^{t}\right) d t}+\frac{(1-\varepsilon)^{3} \int_{n_{1}}^{n} L\left(b^{t}\right) d t}{\int_{0}^{n} L\left(b^{t}\right) d t}<\frac{s_{n}^{2}}{\ell\left(b^{n}\right)}<\frac{\sum_{m=0}^{n_{1}-1} \beta_{m}^{2}}{\int_{0}^{n} L\left(b^{t}\right) d t}+\frac{(1+\varepsilon)^{3} \int_{n_{1}}^{n} L\left(b^{t}\right) d t}{\int_{0}^{n} L\left(b^{t}\right) d t} .
$$

Since $s_{n} \rightarrow \infty$, the inequalities in (4.2) imply that we must also have $\ell\left(b^{n}\right)=\int_{0}^{n} L\left(b^{t}\right) d t \rightarrow \infty$ as $n \uparrow \infty$. It follows that the left- and right-hand sides of $(4.2)$ tend to $(1-\varepsilon)^{3}$ and $(1+\varepsilon)^{3}$ as $n \uparrow \infty$, respectively. Sending $\varepsilon \downarrow 0$ now yields (ii).

(iv) $\Leftrightarrow$ (iii): It follows from the Stolz theorem and its converse given in Lemma 3.1 of [20] that the existence of one of the following limits entails the existence of the other one and that in this case both must be equal:

$$
\lim _{n^{\uparrow} \infty} \frac{s_{n}^{2}}{2^{2 q n} \ell\left(b^{n}\right)} \quad \text { and } \quad \lim _{n^{\uparrow} \infty} \frac{s_{n+1}^{2}-s_{n}^{2}}{2^{2 q(n+1)} \ell\left(b^{n+1}\right)-2^{2 q n} \ell\left(b^{n}\right)} .
$$

Moreover, if it exists, the limit on the right-hand side is equal to

$$
\lim _{n^{\uparrow} \infty} \frac{\beta_{n}^{2}}{2^{2 q n} \ell\left(b^{n}\right)} \cdot \frac{1}{\left(2^{2 q} \frac{\ell\left(b \cdot b^{n}\right)}{\ell\left(b^{n}\right)}-1\right)},
$$

and here the second factor converges to $\left(2^{2 q}-1\right)^{-1}$.

(c): We have

$$
\lim _{n \uparrow \infty} \frac{s_{n-1}^{2}}{s_{n}^{2}}=2^{2 q} \lim _{n \uparrow \infty} \frac{\ell\left(b^{n-1}\right)}{\ell\left(b \cdot b^{n-1}\right)}=2^{2 q} .
$$

This concludes the proof. 
Now we turn toward proving Theorem 3.3 (and also Corollary 3.8 along the way). Following [13, 25], we let $(\Omega, \mathscr{F}, \mathbb{P})$ be a probability space supporting an independent sequence $U_{1}, U_{2}, \cdots$ of symmetric $\{0,1\}$-valued Bernoulli random variables. Then we define the stochastic process $R_{m}:=\sum_{k=1}^{m} U_{k} 2^{k-1}$ and set

$$
Y_{m}:=\frac{\varphi\left(\left(R_{m}+1\right) 2^{-m}\right)-\varphi\left(R_{m} 2^{-m}\right)}{2^{-m}}, \quad \beta_{m}:=2^{m} \alpha_{m}, \quad \text { and } \quad s_{n}^{2}:=\sum_{m=0}^{n-1} \beta_{m}^{2} .
$$

Proposition 3.2 (a) in [25] states that $Y_{1}, Y_{2}, \ldots$ is an i.i.d. sequence of symmetric $\{-1,+1\}$-valued Bernoulli random variables. Moreover, the proof of Theorem 2.1 in [21] gives the same result in the context of Corollary 3.8, so that all subsequent observations are also valid for the functions of the form (3.7).

Note that $R_{m}$ has a uniform distribution on $\left\{0, \ldots, 2^{m}-1\right\}$. Therefore, for $n \in \mathbb{N}$ such that all increments $\left|f\left((k+1) 2^{-n}\right)-f\left(k 2^{-n}\right)\right|$ are less than 1 ,

$$
V_{n}:=\sum_{k=0}^{2^{n}-1} \Phi\left(\left|f\left((k+1) 2^{-n}\right)-f\left(k 2^{-n}\right)\right|\right)=2^{n} \mathbb{E}\left[\Phi\left(\left|f\left(\left(R_{n}+1\right) 2^{-n}\right)-f\left(R_{n} 2^{-n}\right)\right|\right)\right] .
$$

To analyze the expectation on the right, let the $n^{\text {th }}$ truncation of $f$ be given by $f_{n}(t)=\sum_{m=0}^{n-1} \alpha_{m} \varphi\left(2^{m} t\right)$. With the notation introduced in (4.3), we get

$$
\begin{aligned}
f\left(\left(R_{n}+1\right) 2^{-n}\right)-f\left(R_{n} 2^{-n}\right) & =f_{n}\left(\left(R_{n}+1\right) 2^{-n}\right)-f_{n}\left(R_{n} 2^{-n}\right) \\
& =2^{-n} \sum_{m=0}^{n-1} \beta_{m} \frac{\varphi\left(\left(R_{n}+1\right) 2^{m-n}\right)-\varphi\left(R_{n} 2^{m-n}\right)}{2^{m-n}} \\
& =2^{-n} \sum_{m=0}^{n-1} \beta_{m} Y_{n-m},
\end{aligned}
$$

where in the last step we have used that $\varphi\left(x+R_{n} 2^{-m}\right)=\varphi\left(x+R_{m} 2^{-m}\right)$ due to the periodicity of $\varphi$. Hence, if we define

$$
Z_{n}:=\sum_{m=0}^{n-1} \beta_{m} Y_{n-m}=\sum_{m=1}^{n} \beta_{n-m} Y_{m}
$$

then

$$
V_{n}=2^{n} \mathbb{E}\left[\Phi\left(\left|2^{-n} Z_{n}\right|\right)\right]
$$

Thus, the $\Phi$-variation of $f$ is determined by the limit of the right-hand expectations as $n \uparrow \infty$.

Proof of Proposition 3.1. Taking $\Phi(x)=x^{p}$, we get from (4.4) that $V_{n}^{(p)}=2^{n(1-p)} \mathbb{E}\left[\left|Z_{n}\right|^{p}\right]$. By Khintchine's inequality, there exist constants $A_{p}$ and $B_{p}$ only depending on $p$ such that $0<A_{p} \leq B_{p}<\infty$, and

$$
A_{p} 2^{n\left(1-p+p q_{n}\right)}=A_{p} 2^{n(1-p)}\left(s_{n}^{2}\right)^{p / 2} \leq V_{n}^{(p)} \leq B_{p} 2^{n(1-p)}\left(s_{n}^{2}\right)^{p / 2}=B_{p} 2^{n\left(1-p+p q_{n}\right)},
$$

where $q_{n}:=\frac{1}{n} \log _{2} s_{n}$. From here, the assertion is straightforward.

Now we prove Theorem 3.3. For better accessibility, we have divided the proof into several parts.

Proof of Theorem 3.3 (a). Assuming that $\lim _{n} s_{n}^{2}<\infty$, we define $\beta_{m}=\alpha_{m} 2^{m}$ and

$$
\widetilde{Z}_{n}:=\sum_{m=0}^{n-1} \beta_{m} Y_{m}
$$


Then the exchangeability of the sequence $\left\{Y_{m}\right\}_{m \in \mathbb{N}_{0}}$ implies that $Z_{n}$ and $\widetilde{Z}_{n}$ have the same law. Clearly, $\widetilde{Z}_{n} \rightarrow \widetilde{Z}:=\sum_{m=0}^{\infty} \beta_{m} Y_{m}$ in $L^{1}$. Therefore, (4.4) yields for the choice $\Phi(x):=x$,

$$
\sum_{k=0}^{2^{n}-1}\left|f\left((k+1) 2^{-n}\right)-f\left(k 2^{-n}\right)\right|=2^{n} \mathbb{E}\left[\left|2^{-n} Z_{n}\right|\right] \longrightarrow \mathbb{E}[|\widetilde{Z}|]
$$

Since $f$ is continuous, this limit must coincide with the total variation of $f$ (see, e.g., Theorem 2 in $\S 5$ of Chapter VIII in [22]). Conversely, parts (b) and (c) of Theorem 3.3 will imply that $f$ can only be of finite total variation if $\lim _{n} s_{n}^{2}<\infty$.

Proof of Theorem 3.3 (b) for $t=1$. Here, we identify the $\Phi_{0}$-variation $\langle f\rangle_{1}^{\Phi_{0}}$ at time 1 . The linearity of the $\Phi_{0}$-variation $t \mapsto\langle f\rangle_{t}^{\Phi_{0}}$ will be proved subsequently. Condition (3.4) for $q=0$ together with our assumption $s_{n}^{2} \rightarrow \infty$ implies via Proposition 1.5.1 in [3] that $g$ is regularly varying with index $\varrho \geq 0$. Moreover, Remark 3.9, Lemma 2.2, and Proposition 3.10 yield that the law and the first moment of $Z_{n} / s_{n}$ converge, respectively, to $N(0,1)$ and to its first moment.

Let us write $\psi(x):=\sqrt{g(x)}$ so that $2^{n} \Phi_{0}\left(2^{-n}\left|Z_{n}\right|\right)=\left|Z_{n}\right| / \psi\left(n-\log _{2}\left|Z_{n}\right|\right)$. It follows that

$$
2^{n} \Phi_{0}\left(2^{-n}\left|Z_{n}\right|\right) \mathbb{1}_{\left\{\left|Z_{n}\right| / \psi(n) \geq \varepsilon\right\}}=\frac{\psi(n)}{\psi\left(n-\log _{2}\left|Z_{n}\right|\right)} \cdot \frac{\left|Z_{n}\right|}{\psi(n)} \mathbb{1}_{\left\{\left|Z_{n}\right| / \psi(n) \geq \varepsilon\right\}} .
$$

To deal with the first factor on the right, we estimate $n-\log _{2}\left|Z_{n}\right|$. First, on $\left\{\left|Z_{n}\right| / \psi(n) \geq \varepsilon\right\}$, we have $\log _{2}\left|Z_{n}\right| \geq \log _{2} \varepsilon+\ell(n)$, where $\ell(x):=\log _{2} \psi(x)$ is slowly varying by Proposition 1.5.7 in [3] and hence satisfies $\ell(n) / n \rightarrow 0$ according to Proposition 1.3.6 (v) in [3]. Therefore, there exists $n_{3} \in \mathbb{N}$ such that

$$
n-\log _{2}\left|Z_{n}\right| \leq n-\log _{2} \varepsilon-\ell(n) \leq(1+\varepsilon) n \quad \text { on }\left\{\left|Z_{n}\right| / \psi(n) \geq \varepsilon\right\} \text { for all } n \geq n_{3} .
$$

Second, to get a lower bound, we use that $\left|Z_{n}\right| \leq \sum_{m=0}^{n-1}\left|\beta_{m}\right|$. Jensen's inequality gives furthermore that

$$
\left|Z_{n}\right| \leq \sum_{m=0}^{n-1}\left|\beta_{m}\right| \leq n \cdot \sqrt{\frac{1}{n} \sum_{m=0}^{n-1} \beta_{m}^{2}}=\sqrt{n} s_{n}
$$

By (3.4), there is $n_{4} \in \mathbb{N}$ such that $s_{n} \leq(1+\varepsilon) \psi(n)$ for all $n \geq n_{4}$. Thus, there is $n_{5} \geq n_{4}$ such that

$$
n-\log _{2}\left|Z_{n}\right| \geq n-\log _{2}(1+\varepsilon)-\frac{1}{2} \log _{2} n-\ell(n) \geq(1-\varepsilon) n \quad \text { for all } n \geq n_{5} .
$$

Combining (4.6) and (4.8) now yields

$$
\frac{\psi(n)}{\psi\left(n-\log _{2}\left|Z_{n}\right|\right)} \geq \inf _{1-\varepsilon \leq \lambda \leq 1+\varepsilon} \frac{\psi(n)}{\psi(\lambda n)} \quad \text { for } n \geq n_{5} \text { on }\left\{\left|Z_{n}\right| / \psi(n) \geq \varepsilon\right\} .
$$

According to the uniform convergence theorem for regularly varying functions (Theorem 1.5.2 in [3]) and the fact that $\psi$ is regularly varying with index $\varrho / 2 \geq 0$ by Proposition 1.5 .7 (i) in [3], we have

$$
\inf _{1-\varepsilon \leq \lambda \leq 1+\varepsilon} \frac{\psi(n)}{\psi(\lambda n)} \longrightarrow(1-\varepsilon)^{\varrho / 2} .
$$

To deal with the second factor on the right-hand side of $(4.5)$, we choose $n_{6} \geq n_{5}$ such that $\psi(n) \leq$ $(1+\varepsilon) s_{n}$ for all $n \geq n_{6}$. Then, for $n \geq n_{6}$,

$$
\frac{\left|Z_{n}\right|}{\psi(n)} \mathbb{1}_{\left\{\left|Z_{n}\right| / \psi(n) \geq \varepsilon\right\}} \geq \frac{\left|Z_{n}\right|}{(1+\varepsilon) s_{n}} \mathbb{1}_{\left\{\left|Z_{n}\right| / s_{n} \geq \varepsilon(1+\varepsilon)\right\}} .
$$


Altogether, we get

$$
\liminf _{n \uparrow \infty} 2^{n} \mathbb{E}\left[\Phi_{0}\left(2^{-n}\left|Z_{n}\right|\right)\right] \geq \frac{(1-\varepsilon)^{\varrho / 2}}{(1+\varepsilon) \sqrt{2 \pi}} \int_{\{|z| \geq \varepsilon(1+\varepsilon)\}}|z| e^{-z^{2} / 2} d z .
$$

With (4.4), we thus get $\lim \inf _{n} V_{n} \geq \sqrt{2 / \pi}$ by sending $\varepsilon \downarrow 0$.

To get an upper bound, we recall that $x \mapsto \Phi_{0}(1 / x)$ is regularly varying (at infinity) with index $-1<0$. Theorem 1.8.2 in [3] hence yields a function $h$ that is regularly varying with index -1 , strictly decreasing, and satisfies $\Phi_{0}(1 / x) \leq h(x)$ as well as $h(x) / \Phi_{0}(1 / x) \rightarrow 1$ as $x \uparrow \infty$. Thus, the function $\Psi(x):=h(1 / x)$ is strictly increasing, regularly varying at zero with index 1 , and satisfies $\Phi_{0} \leq \Psi$ as well as $\Psi(x) / \Phi_{0}(x) \rightarrow 1$ as $x \downarrow 0$. Let $\varepsilon>0$ be given and choose $x_{0}>0$ such that $\Psi(x) \leq \Phi_{0}(x) /(1-\varepsilon)$ for all $x \leq x_{0}$. By (4.7), there is $n_{7} \in \mathbb{N}$ such that $2^{-n}\left|Z_{n}\right| \leq x_{0}$ for all $n \geq n_{7}$. For such $n$, we get

$$
\begin{aligned}
2^{n} \mathbb{E}\left[\Phi_{0}\left(2^{-n}\left|Z_{n}\right|\right)\right] & \leq 2^{n} \mathbb{E}\left[\Psi\left(2^{-n}\left|Z_{n}\right|\right)\right] \leq 2^{n} \mathbb{E}\left[\Psi\left(2^{-n}\left(\varepsilon+\left|Z_{n}\right|\right)\right)\right] \leq \frac{2^{n}}{1-\varepsilon} \mathbb{E}\left[\Phi_{0}\left(2^{-n}\left(\varepsilon+\left|Z_{n}\right|\right)\right)\right] \\
& =\frac{s_{n}}{(1-\varepsilon) \psi(n)} \mathbb{E}\left[\frac{\varepsilon+\left|Z_{n}\right|}{s_{n}} \cdot \frac{\psi(n)}{\psi\left(n-\log _{2}\left(\varepsilon+\left|Z_{n}\right|\right)\right)}\right] .
\end{aligned}
$$

Clearly, the factor $s_{n} / \psi(n)$ converges to 1 . Moreover, by (4.8) there is $n_{8} \geq n_{5} \vee n_{7}$ such that

$$
(1-\varepsilon) n \leq n-\log _{2}\left(\varepsilon+\left|Z_{n}\right|\right) \leq n-\log _{2} \varepsilon \leq(1+\varepsilon) n \quad \text { for all } n \geq n_{8} .
$$

As in (4.9) and (4.10), we thus get

$$
\frac{\psi(n)}{\psi\left(n-\log _{2}\left(\varepsilon+\left|Z_{n}\right|\right)\right)} \leq \sup _{1-\varepsilon \leq \lambda \leq 1+\varepsilon} \frac{\psi(n)}{\psi(\lambda n)} \longrightarrow(1+\varepsilon)^{\varrho / 2} .
$$

We hence obtain the upper bound

$$
\limsup _{n \uparrow \infty} V_{n} \leq \frac{(1+\varepsilon)^{\varrho / 2}}{(1-\varepsilon) \sqrt{2 \pi}} \int|z| e^{-z^{2} / 2} d z
$$

where the right-hand side reduces to $\sqrt{2 / \pi}$ as $\varepsilon \downarrow 0$.

The proof of Theorem 3.3 (b) for $0<t<1$ requires that we truncate the summation for $V_{n}$ in $(4.4)$ at those indices $k$ for which $k 2^{-n} \leq t$. This is equivalent to restricting the expectation in (4.4) to the set $\left\{2^{-n} R_{n} \leq t\right\}$. The following proof adapts the arguments of the proof of Theorem 3.3 (b) for $t=1$ to this restricted case.

Proof of Theorem 3.3 (b) for $0<t<1$. Let $t \in(0,1)$ be given. The $\Phi_{0}$-variation of $f$ over the interval $[0, t]$ is equal to $\lim _{n} V_{n, t}$, where

$$
V_{n, t}:=\sum_{k=0}^{2^{n}-1} \Phi_{0}\left(\left|f\left((k+1) 2^{-n}\right)-f\left(k 2^{-n}\right)\right|\right) \mathbb{1}_{[0, t]}\left(k 2^{-n}\right)=2^{n} \mathbb{E}\left[\Phi_{0}\left(2^{-n}\left|Z_{n}\right|\right) \mathbb{1}_{\left\{2^{-n} R_{n} \leq t\right\}}\right] .
$$

This can be proved similarly as in the derivation of (4.4), see e.g. [13]. We fix $\delta \in(0, t \wedge(1-t))$ and $m \in \mathbb{N}$ that $2^{-m} \leq \delta$. Recall that $R_{m}:=\sum_{k=1}^{m} U_{k} 2^{k-1}$ and let us denote $R_{m, n}:=R_{n}-R_{n-m}$ for short. Then

$$
\left\{2^{-n} R_{m, n} \leq t-\delta\right\} \subseteq\left\{2^{-n} R_{n} \leq t\right\} \subseteq\left\{2^{-n} R_{m, n} \leq t\right\}
$$


First, we derive a lower bound for $V_{n, t}$. To this end, we define for $0 \leq m<n$,

$$
Z_{m, n}:=\sum_{k=1}^{n-m} \beta_{n-k} Y_{k}=Z_{n}-\sum_{k=n-m+1}^{n} \beta_{n-k} Y_{k}
$$

Then, since $s_{n} \rightarrow \infty$,

$$
\frac{\left|Z_{n}-Z_{m, n}\right|}{s_{n}} \leq \frac{1}{s_{n}} \sum_{k=0}^{m-1}\left|\beta_{k}\right|=: a_{n} \longrightarrow 0,
$$

and so the laws of $Z_{m, n} / s_{n}$ converge to $N(0,1)$ in the $L^{1}$-Wasserstein distance by Slutsky's theorem. By combining (4.5), (4.9), (4.10), and (4.11), there is $n_{9} \geq n_{6} \vee m$ such that for all $n \geq n_{9}$, with $c_{\varepsilon}:=(1-\varepsilon)^{1+\varrho / 2} /(1+\varepsilon)$,

$$
\begin{aligned}
2^{n} \Phi_{0}\left(2^{-n}\left|Z_{n}\right|\right) \mathbb{1}_{\left\{\left|Z_{n}\right| / \psi(n) \geq \varepsilon, 2^{-n} R_{n} \leq t\right\}} & \geq \frac{c_{\varepsilon}\left|Z_{n}\right|}{s_{n}} \mathbb{1}_{\left\{\left|Z_{n}\right| / \psi(n) \geq \varepsilon, 2^{-n} R_{n} \leq t\right\}} \\
& \geq \frac{c_{\varepsilon}\left|Z_{m, n}\right|}{s_{n}} \mathbb{1}_{\left\{\left|Z_{n}\right| / \psi(n) \geq \varepsilon, 2^{-n} R_{n} \leq t\right\}}-\frac{c_{\varepsilon}\left|Z_{n}-Z_{m, n}\right|}{s_{n}} \\
& \geq \frac{c_{\varepsilon}\left|Z_{m, n}\right|}{s_{n}} \mathbb{1}_{\left\{\left|Z_{n}\right| / \psi(n) \geq \varepsilon, 2^{-n} R_{n} \leq t\right\}}-\frac{c_{\varepsilon} \sum_{k=0}^{m-1}\left|\beta_{k}\right|}{s_{n}},
\end{aligned}
$$

where the last two inequalities hold due to the triangle inequality. Again, the right-most term decays to zero as $n \uparrow \infty$. Hence, there is $n_{10} \geq n_{9}$ such that for $n \geq n_{10}$ and $\left|Z_{m, n}\right| / s_{n} \geq 2 \varepsilon(1+\varepsilon)$,

$$
\frac{\left|Z_{n}\right|}{s_{n}} \geq \frac{\left|Z_{m, n}\right|-\left|Z_{n}-Z_{m, n}\right|}{s_{n}} \geq \frac{\left|Z_{m, n}\right|-\sum_{k=0}^{m-1}\left|\beta_{k}\right|}{s_{n}} \geq \varepsilon(1+\varepsilon) .
$$

Hence, for those $n$ and $m$, we have $\left\{\left|Z_{m, n}\right| / s_{n} \geq 2 \varepsilon(1+\varepsilon)\right\} \subseteq\left\{\left|Z_{n}\right| / s_{n} \geq \varepsilon(1+\varepsilon)\right\}$ and in turn

$$
\begin{aligned}
V_{n, t}+c_{\varepsilon} a_{n} & \geq \mathbb{E}\left[\frac{c_{\varepsilon}\left|Z_{m, n}\right|}{s_{n}} \mathbb{1}_{\left\{\left|Z_{n}\right| / \psi(n) \geq \varepsilon, 2^{-n} R_{n} \leq t\right\}}\right] \geq \mathbb{E}\left[\frac{c_{\varepsilon}\left|Z_{m, n}\right|}{s_{n}} \mathbb{1}_{\left\{\left|Z_{m, n}\right| / s_{n} \geq 2 \varepsilon(1+\varepsilon), 2^{-n} R_{m, n} \leq t-\delta\right\}}\right] \\
& \geq \mathbb{E}\left[\frac{c_{\varepsilon}\left|Z_{m, n}\right|}{s_{n}} \mathbb{1}_{\left\{\left|Z_{m, n}\right| / s_{n} \geq 2 \varepsilon(1+\varepsilon)\right\}}\right] \cdot \mathbb{P}\left[2^{-n} R_{n} \leq t-\delta\right],
\end{aligned}
$$

where the last step follows from (4.13) and the independence of $Z_{m, n}$ and $R_{m, n}$. Clearly, $\mathbb{P}\left[2^{-n} R_{n} \leq\right.$ $t-\delta] \rightarrow t-\delta$ and

$$
\mathbb{E}\left[\frac{c_{\varepsilon}\left|Z_{m, n}\right|}{s_{n}} \mathbb{1}_{\left\{\left|Z_{m, n}\right| / s_{n} \geq 2 \varepsilon(1+\varepsilon)\right\}}\right] \longrightarrow \frac{c_{\varepsilon}}{\sqrt{2 \pi}} \int_{\{|z| \geq 2 \varepsilon(1+\varepsilon)\}}|z| e^{-z^{2} / 2} d z,
$$

since $s_{n-m} / s_{n} \rightarrow 1$ by Proposition 3.10. Hence

$$
\liminf _{n \uparrow \infty} V_{n, t} \geq \frac{c_{\varepsilon}(t-\delta)}{\sqrt{2 \pi}} \int_{\{|z| \geq 2 \varepsilon(1+\varepsilon)\}}|z| e^{-z^{2} / 2} d z .
$$

To obtain a corresponding upper bound, we can argue exactly as in the derivation of (4.12) to get

$$
\limsup _{n \uparrow \infty} V_{n, t} \leq(1+\varepsilon)^{\varrho / 2} \limsup _{n \uparrow \infty} \mathbb{E}\left[\frac{\varepsilon+\left|Z_{n}\right|}{s_{n}} \mathbb{1}_{\left\{2^{-n} R_{m, n} \leq t\right\}}\right]=(1+\varepsilon)^{\varrho / 2} \limsup _{n \uparrow \infty} \mathbb{E}\left[\frac{\left|Z_{n}\right|}{s_{n}} \mathbb{1}_{\left\{2^{-n} R_{m, n} \leq t\right\}}\right] .
$$

Using again the independence of $Z_{m, n}$ and $R_{m, n}$, we find that

$$
\mathbb{E}\left[\left|Z_{n}\right| \mathbb{1}_{\left\{2^{-n} R_{m, n} \leq t\right\}}\right] \leq \mathbb{E}\left[\left|Z_{m, n}\right|\right] \cdot \mathbb{P}\left[2^{-n} R_{m, n} \leq t\right]+\sum_{k=0}^{m-1}\left|\beta_{k}\right|
$$


By (4.13), we have $\mathbb{P}\left[2^{-n} R_{m, n} \leq t\right] \leq \mathbb{P}\left[2^{-n} R_{n} \leq t+\delta\right] \rightarrow t+\delta$. Recall that $\frac{1}{s_{n}} \sum_{k=0}^{m-1}\left|\beta_{k}\right| \rightarrow 0$ as $n \uparrow \infty$. Next, the function $\ell(x):=g\left(\log _{2} x\right)$ is slowly varying by Remark 3.9 , and so

$$
\frac{g(n)}{g(n-m)}=\frac{\ell\left(2^{n}\right)}{\ell\left(2^{-m} 2^{n}\right)} \longrightarrow 1 \quad \text { as } n \uparrow \infty .
$$

Thus, our condition (3.4) for $q=0$ implies that

$$
\mathbb{E}\left[\frac{\left|Z_{m, n}\right|}{s_{n}}\right]=\frac{s_{n-m}}{s_{n}} \mathbb{E}\left[\frac{\left|Z_{m, n}\right|}{s_{n-m}}\right] \longrightarrow \sqrt{\frac{2}{\pi}} \quad \text { as } n \uparrow \infty .
$$

Altogether, we conclude that $\lim _{\sup _{n}} V_{n, t} \leq(1+\varepsilon)^{\varrho / 2}(t+\delta) \sqrt{2 / \pi}$. Combining this inequality with (4.14) and sending $\varepsilon$ and $\delta$ to zero, we conclude the proof of the linearity of the $\Phi_{0}$-variation.

The proof of part (c) of Theorem 3.3 will be prepared with the following two lemmas.

Lemma 4.1. Under the conditions of Theorem 3.3 (c), there is a constant $K$ such that for all $\omega \in \Omega$ and all sufficiently large $n \in \mathbb{N}$,

$$
2^{-q n}\left|Z_{n}(\omega)\right| \leq K \sqrt{g(n)} \quad \text { and } \quad\left|Z_{n}(\omega)\right| \leq K s_{n} .
$$

Proof. The function $\ell(x):=g\left(\log _{2} x\right)$ is slowly varying by Remark 3.9, and so $\ell$ and the sequence $\left\{\beta_{m}\right\}_{m \in \mathbb{N}_{0}}$ satisfy the conditions of Theorem 2.1. Recall from (2.8) in the proof of that theorem that there exists $\delta_{1}>0$ such that for every $\delta \in\left(0, \delta_{1}\right)$ there is $n_{1} \in \mathbb{N}$ such that for all $m \geq n_{1}$,

$$
2^{q m} \sqrt{(1-\delta) \ell\left(2^{m}\right)-(1+\delta) \ell\left(2^{m-1}\right) 2^{-2 q}}<\left|\beta_{m}\right|<2^{q m} \sqrt{(1+\delta) \ell\left(2^{m}\right)-(1-\delta) \ell\left(2^{m-1}\right) 2^{-2 q}},
$$

and the arguments of the two square roots are positive. Now let $\delta \in\left(0, \delta_{1}\right)$ be given and take $n_{2} \geq n_{1}$ such that $1-\delta<g(n) / g(n-1)<1+\delta$ for any $n \geq n_{2}$; this is possible by (4.15). Since $g$ is regularly varying, we have $2^{2 q n} g(n) \rightarrow \infty$ as $n \uparrow \infty$ (see, e.g., Proposition B.1.9 in [5]). Therefore, we may apply the Stolz-Cesáro theorem in its general form so as to obtain

$$
\begin{aligned}
\limsup _{n \uparrow \infty} \frac{\sum_{m=0}^{n}\left|\beta_{m}\right|}{2^{q n} \sqrt{g(n)}} & \leq \limsup _{n \uparrow \infty} \frac{\left|\beta_{n}\right|}{2^{q n} \sqrt{g(n)}-2^{q(n-1)} \sqrt{g(n-1)}} \\
& \leq \limsup _{n \uparrow \infty} \frac{\sqrt{(1+\delta) g(n)-(1-\delta) g(n-1) 2^{-2 q}}}{\sqrt{g(n)}-\sqrt{2^{-2 q} g(n-1)}} \\
& \leq \frac{\sqrt{(1+\delta)^{2} 2^{2 q}-(1-\delta)}}{\sqrt{2^{2 q}(1-\delta)}-1}
\end{aligned}
$$

Since $\left|Z_{n}\right| \leq \sum_{m=0}^{n-1}\left|\beta_{m}\right|$, the claim follows.

Lemma 4.2. Suppose that $\left\{z_{n}\right\}_{n \in \mathbb{N}_{0}}$ is a sequence of nonnegative numbers converging to $z>0$. Then $2^{n} \Phi_{q}\left(2^{-n} s_{n} z_{n}\right) \rightarrow z^{1 /(1-q)}$ as $n \uparrow \infty$.

Proof. We may assume without loss of generality that $z_{n}>0$ for all $n$. Then we may write

$$
\begin{aligned}
2^{n} \Phi_{q}\left(2^{-n} s_{n} z_{n}\right) & =2^{n}\left(2^{-n} s_{n} z_{n}\right)^{1 /(1-q)} g\left(\frac{n-\log _{2} s_{n}-\log _{2} z_{n}}{1-q}\right)^{-1 /(2(1-q))} \\
& =\left(\frac{s_{n}}{2^{q n} \sqrt{g(n)}}\right)^{1 /(1-q)} z_{n}^{1 /(1-q)}\left(\frac{g(n)}{g\left(\frac{n-\log _{2} s_{n}-\log _{2} z_{n}}{1-q}\right)}\right)^{1 /(2(1-q))}
\end{aligned}
$$


By (3.4), the first factor on the right converges to 1 . The second factor converges to $z^{1 /(1-q)}$ by assumption. To deal with the third factor, (3.4) implies that there is $n_{0} \in \mathbb{N}$ such that

$$
q n-\log _{2} \sqrt{g(n)}-1 \leq \log _{2} s_{n} \leq q n-\log _{2} \sqrt{g(n)}+1 \quad \text { for all } n \geq n_{0} .
$$

Theorem 1.4.1 and Proposition 1.3.6 (i) in [3] give moreover that there exists $\kappa^{+}, \kappa^{-} \in \mathbb{R}$ such that $\kappa^{-} \log _{2} n \leq \log _{2} \sqrt{g(n)} \leq \kappa^{+} \log _{2} n$. Thus, if $n \geq n_{0}$ is sufficiently large such that $z / 2 \leq z_{n} \leq 2 z$, then

$$
n+\frac{-1-\log _{2}(2 z)-\kappa^{+} \log _{2} n}{1-q} \leq \frac{n-\log _{2} s_{n}-\log _{2} z_{n}}{1-q} \leq n+\frac{1-\log _{2}(z / 2)-\kappa^{-} \log _{2} n}{1-q} .
$$

For sufficiently large $n$, the center term can thus be expressed as $n \lambda_{n}$, where $\lambda_{n} \in[1 / 2,3 / 2]$ and $\lambda_{n} \rightarrow 1$. The uniform convergence theorem for regularly varying functions (e.g., Theorem 1.5.2 in [3]) hence implies that

$$
\frac{g(n)}{g\left(\frac{n-\log _{2} s_{n}-\log _{2} z_{n}}{1-q}\right)} \longrightarrow 1
$$

as $n \uparrow \infty$. This concludes the proof.

Proof of Theorem 3.3 (c). We prove part (c) only for $t=1$. The extension to $0<t<1$ is almost verbatim identical to the one in part (b) and hence left to the reader. We write

$$
\widehat{Z}_{n}:=\frac{1}{s_{n}} \sum_{m=1}^{n}\left|\beta_{n-m}\right| Y_{m}
$$

Then the law of $2^{n} \Phi_{q}\left(2^{-n}\left|Z_{n}\right|\right)$ is the same as that of $2^{n} \Phi_{q}\left(2^{-n} s_{n}\left|\widehat{Z}_{n}\right|\right)$. By Theorem 2.1, we have $\left|\widehat{Z}_{n}\right| \rightarrow|Z|$ in $L^{\infty}$. Moreover, by either Corollary 6.6 or Remark 6.7 in [6], the law of $Z$ has no atoms. In particular, we have $\mathbb{P}[Z=0]=0$. Lemma 4.2 hence yields that $\mathbb{P}$-a.s. $2^{n} \Phi_{q}\left(2^{-n} s_{n}\left|\widehat{Z}_{n}\right|\right) \rightarrow|Z|^{1 /(1-q)}$. Fatou's lemma thus gives immediately that

$$
\liminf _{n \uparrow \infty} V_{n}=\liminf _{n \uparrow \infty} 2^{n} \mathbb{E}\left[\Phi_{q}\left(2^{-n}\left|Z_{n}\right|\right)\right]=\liminf _{n \uparrow \infty} \mathbb{E}\left[2^{n} \Phi_{q}\left(2^{-n} s_{n}\left|\widehat{Z}_{n}\right|\right)\right] \geq \mathbb{E}\left[|Z|^{1 /(1-q)}\right] .
$$

To get an upper bound, we argue as in the proof of Theorem 3.3 (b) for $t=1$ that there exists an increasing function $\Psi$ that is regularly varying at zero with index $1 /(1-q)$ and satisfies $\Phi_{q}(x) \leq \Psi(x)$ for all sufficiently small $x$ as well as $\Psi(x) / \Phi_{q}(x) \rightarrow 1$ as $x \downarrow 0$. In particular, we have $2^{n} \Psi\left(2^{-n} s_{n} z_{n}\right) \rightarrow$ $z^{1 /(1-q)}$ if $\left\{z_{n}\right\}_{n \in \mathbb{N}_{0}}$ is a sequence of nonnegative numbers converging to $z>0$. Let $\varepsilon>0$ be given. We choose $x_{0}>0$ such that $\Psi(x) \leq \Phi_{q}(x) /(1-\varepsilon)$ for all $x \leq x_{0}$. Since the sequence $\left\{\widehat{Z}_{n}\right\}$ is uniformly bounded, there is $n_{0} \in \mathbb{N}$ such that $2^{-n} s_{n}\left(\varepsilon+\left|\widehat{Z}_{n}\right|\right) \leq x_{0} \mathbb{P}$-a.s. for all $n \geq n_{0}$. For such $n$, we hence have

$V_{n}=2^{n} \mathbb{E}\left[\Phi_{q}\left(2^{-n} s_{n}\left|\widehat{Z}_{n}\right|\right)\right] \leq 2^{n} \mathbb{E}\left[\Psi\left(2^{-n} s_{n}\left|\widehat{Z}_{n}\right|\right)\right] \leq 2^{n} \mathbb{E}\left[\Psi\left(2^{-n} s_{n}\left(\varepsilon+\left|\widehat{Z}_{n}\right|\right)\right)\right] \leq \frac{2^{n}}{1-\varepsilon} \mathbb{E}\left[\Phi_{q}\left(2^{-n} s_{n}\left(\varepsilon+\left|\widehat{Z}_{n}\right|\right)\right)\right]$.

Moreover, we see from (4.16) that there exists $n_{1} \geq n_{0}$ such that the random variables $\Lambda_{n}$ defined through

$$
\Lambda_{n}:=\frac{n-\log _{2} s_{n}-\log _{2}\left(\varepsilon+\left|\widehat{Z}_{n}\right|\right)}{n(1-q)}
$$

take values in $[1 / 2,3 / 2]$ for $n \geq n_{1}$ and satisfy $\Lambda_{n} \rightarrow 1 \mathbb{P}$-a.s. Hence, with $\varrho$ denoting the index of regular variation of $g$,

$$
0 \leq \frac{g(n)}{g\left(\frac{n-\log _{2} s_{n}-\log _{2}\left(\varepsilon+\left|\widehat{Z}_{n}\right|\right)}{1-q}\right)}=\frac{g(n)}{g\left(n \Lambda_{n}\right)} \leq \sup _{1 / 2 \leq \lambda \leq 3 / 2} \frac{g(n)}{g(n \lambda)} \longrightarrow\left(\frac{3}{2}\right)^{\varrho}
$$


according to the uniform convergence theorem for regularly varying functions (e.g., Theorem 1.5.2 in [3]). Hence, Lemma 4.2 and dominated convergence give that

$$
\limsup _{n \uparrow \infty} V_{n} \leq \limsup _{n \uparrow \infty} \frac{1}{1-\varepsilon} 2^{n} \mathbb{E}\left[\Phi_{q}\left(2^{-n} s_{n}\left(\varepsilon+\left|\widehat{Z}_{n}\right|\right)\right)\right]=\frac{1}{1-\varepsilon} \mathbb{E}\left[(\varepsilon+|Z|)^{1 /(1-q)}\right] .
$$

Sending $\varepsilon \downarrow 0$ gives the desired upper bound.

Proof of Corollary 3.5. (a) Taking logarithms in (3.4) and using the fact that $\frac{1}{n} \log _{2} g(n) \rightarrow 0$, we get $\frac{1}{n} \log _{2} s_{n} \rightarrow q$ as $n \uparrow \infty$. Hence, the result follows from Proposition 3.1.

(b) If $0<c<\infty$, then (3.4) holds also if $g$ is replaced with $\widetilde{g}(x):=c$. Hence, the assertion follows immediately from Theorem 3.3. If $c=0$, then for any $\varepsilon>0$ there is $\delta>0$ such that

$$
g\left(\frac{-\log _{2} \eta}{1-q}\right)<\varepsilon \quad \text { for all } \eta<\delta
$$

Now it suffices to take $n_{0} \in \mathbb{N}$ such that $\left|f\left((k+1) 2^{-n}\right)-f\left(k 2^{-n}\right)\right|<\delta$ for all $k$ and $n \geq n_{0}$ to obtain that

$$
\sum_{k=0}^{\left\lfloor t 2^{n}\right\rfloor}\left|f\left((k+1) 2^{-n}\right)-f\left(k 2^{-n}\right)\right|^{p} \leq \varepsilon^{p / 2} \sum_{k=0}^{\left\lfloor t 2^{n}\right\rfloor} \Phi_{q}\left(\left|f\left((k+1) 2^{-n}\right)-f\left(k 2^{-n}\right)\right|\right) .
$$

Sending $\varepsilon \downarrow 0$ gives the result. The case $c=\infty$ is obtained analogously.

\section{References}

[1] Pieter C. Allaart. On a flexible class of continuous functions with uniform local structure. Journal of the Mathematical Society of Japan, 61(1):237-262, 2009.

[2] Pieter C. Allaart and Kiko Kawamura. The Takagi function: a survey. Real Analysis Exchange, 37(1):1-54, 2011.

[3] N. H. Bingham, C. M. Goldie, and J. L. Teugels. Regular variation, volume 27 of Encyclopedia of Mathematics and its Applications. Cambridge University Press, Cambridge, 1989.

[4] Rama Cont and Nicolas Perkowski. Pathwise integration and change of variable formulas for continuous paths with arbitrary regularity. Trans. Amer. Math. Soc. Ser. B, 6:161-186, 2019.

[5] Laurens de Haan and Ana Ferreira. Extreme value theory. Springer Series in Operations Research and Financial Engineering. Springer, New York, 2006. An introduction.

[6] Dorin Ervin Dutkay and Palle E. T. Jorgensen. Harmonic analysis and dynamics for affine iterated function systems. Houston J. Math., 33(3):877-905, 2007.

[7] G. Faber. Einfaches Beispiel einer stetigen nirgends differenzierbaren Funktion. Jahresber. Dtsch. Math.-Ver., 16:538-540, 1907.

[8] Hans. Föllmer. Calcul d'Itô sans probabilités. In Seminar on Probability, XV (Univ. Strasbourg, Strasbourg, 1979/1980), volume 850 of Lecture Notes in Math., pages 143-150. Springer, Berlin, 1981.

[9] Hans Föllmer and Alexander Schied. Stochastic finance. An introduction in discrete time. De Gruyter Graduate. De Gruyter, Berlin, fourth revised and extended edition, 2016. 
[10] Nina Gantert. Self-similarity of Brownian motion and a large deviation principle for random fields on a binary tree. Probab. Theory Related Fields, 98(1):7-20, 1994.

[11] Jim Gatheral, Thibault Jaisson, and Mathieu Rosenbaum. Volatility is rough. Quantitative Finance, 18(6):933-949, 2018.

[12] E. G. Gladyshev. A new limit theorem for stochastic processes with Gaussian increments. Teor. Verojatnost. i Primenen, 6:57-66, 1961.

[13] Xiyue Han, Alexander Schied, and Zhenyuan Zhang. A probabilistic approach to the $\Phi$-variation of classical fractal functions with critical roughness. Statist. Probab. Lett., 168:108920, 2021.

[14] Masayoshi Hata and Masaya Yamaguti. The Takagi function and its generalization. Japan J. Appl. Math., 1(1):183-199, 1984.

[15] Takayuki Kawada and Norio Kôno. On the variation of Gaussian processes. In Proceedings of the Second Japan-USSR Symposium on Probability Theory (Kyoto, 1972), pages 176-192. Lecture Notes in Math., Vol. 330, 1973.

[16] Norio Kôno. Oscillation of sample functions in stationary Gaussian processes. Osaka Math. J., 6:1-12, 1969.

[17] Norio Kôno. On generalized Takagi functions. Acta Math. Hungar., 49(3-4):315-324, 1987.

[18] Michael B. Marcus and Jay Rosen. $\Phi$-variation of the local times of symmetric Lévy processes and stationary Gaussian processes. In Seminar on Stochastic Processes, 1992 (Seattle, WA, 1992), volume 33 of Progr. Probab., pages 209-220. Birkhäuser Boston, Boston, MA, 1993.

[19] Michael B. Marcus and Jay Rosen. Markov processes, Gaussian processes, and local times, volume 100 of Cambridge Studies in Advanced Mathematics. Cambridge University Press, Cambridge, 2006 .

[20] Yuliya Mishura and Alexander Schied. Constructing functions with prescribed pathwise quadratic variation. J. Math. Anal. Appl., 442(1):117-137, 2016.

[21] Yuliya Mishura and Alexander Schied. On (signed) Takagi-Landsberg functions: $p$ th variation, maximum, and modulus of continuity. J. Math. Anal. Appl., 473(1):258-272, 2019.

[22] I. P. Natanson. Theory of functions of a real variable. Frederick Ungar Publishing Co., New York, 1955. Translated by Leo F. Boron with the collaboration of Edwin Hewitt.

[23] Yuval Peres, Wilhelm Schlag, and Boris Solomyak. Sixty years of Bernoulli convolutions. In Fractal geometry and stochastics, II (Greifswald/Koserow, 1998), volume 46 of Progr. Probab., pages 39-65. Birkhäuser, Basel, 2000.

[24] Alexander Schied. On a class of generalized Takagi functions with linear pathwise quadratic variation. J. Math. Anal. Appl., 433:974-990, 2016.

[25] Alexander Schied and Zhenyuan Zhang. On the $p$ th variation of a class of fractal functions. Proc. Amer. Math. Soc., 148(12):5399-5412, 2020.

[26] Teiji Takagi. A simple example of the continuous function without derivative. In Proc. Phys. Math. Soc. Japan, volume 1, pages 176-177, 1903. 
[27] S. J. Taylor. Exact asymptotic estimates of Brownian path variation. Duke Math. J., 39:219-241, 1972

[28] Ou Zhao, Michael Woodroofe, and Dalibor Volný. A central limit theorem for reversible processes with nonlinear growth of variance. J. Appl. Probab., 47(4):1195-1202, 2010. 